\title{
Radial velocity confirmation of Kepler-91 b
}

\section{Additional evidence of its planetary nature using the Calar Alto/CAFE instrument}

\author{
J. Lillo-Box ${ }^{1}$, D. Barrado ${ }^{1}$, Th. Henning ${ }^{2}$, L. Mancini ${ }^{2}$, S. Ciceri ${ }^{2}$, P. Figueira ${ }^{3}$, \\ N. C. Santos ${ }^{3,4}$, J. Aceituno ${ }^{5}$, and S. Sánchez ${ }^{6}$
}

\author{
1 Depto. de Astrofísica, Centro de Astrobiología (CSIC-INTA), ESAC campus 28691 Villanueva de la Cañada (Madrid), Spain \\ e-mail: Jorge.Lillo@cab.inta-csic.es \\ 2 Max Planck Institute for Astronomy, Königstuhl 17, 69117 Heidelberg, Germany \\ 3 Centro de Astrofísica, Universidade do Porto, Rua das Estrelas, 4150-762 Porto, Portugal \\ 4 Departamento de Física e Astronomia, Faculdade de Ciências, Universidade do Porto, 4150-762 Porto, Portugal \\ 5 Centro Astronómico Hispano-Alemán (CAHA), Calar Alto Observatory, c/ Jesús Durbán Remón 2-2, 04004 Almería, Spain \\ ${ }^{6}$ Instituto de Astronomía, Universidad Nacional Autonóma de México, A.P. 70-264, 04510 México, D.F, Mexico \\ Received 11 July 2014 / Accepted 31 July 2014
}

\section{ABSTRACT}

\begin{abstract}
The object transiting the star Kepler-91 was recently assessed as being of planetary nature. The confirmation was achieved by analysing the light-curve modulations observed in the Kepler data. However, quasi-simultaneous studies claimed a self-luminous nature for this object, thus rejecting it as a planet. In this work, we apply an independent approach to confirm the planetary mass of Kepler-91b by using multi-epoch high-resolution spectroscopy obtained with the Calar Alto Fiber-fed Echelle spectrograph (CAFE). We obtain the physical and orbital parameters with the radial velocity technique. In particular, we derive a value of $1.09 \pm 0.20 M_{\text {Jup }}$ for the mass of Kepler-91b, in excellent agreement with our previous estimate that was based on the orbital brightness modulation.
\end{abstract}

Key words. planets and satellites: gaseous planets - techniques: radial velocities - planets and satellites: individual: Kepler-91 planets and satellites: individual: KOI-2133 - planets and satellites: individual: KIC 8219268 - planet-star interactions

\section{Introduction}

Several techniques, some previously applied to binary systems, have been recently improved to obtain the mass of planetarysize objects. They include radial velocity (RV) measurements (Mayor \& Queloz 1995; Santerne et al. 2012), accurate astrometry (Muterspaugh et al. 2010; Lazorenko et al. 2011), microlensing (Mao \& Paczynski 1991; Gould \& Loeb 1992), transit-timing variations (e.g. Holman et al. 2010), or light-curve modulations (e.g. Borucki et al. 2009). Because of its easy applicability to a wide range of masses, the RV method has been the most popular in the past two decades. Currently, thanks to the unprecedented accurate photometry obtained by the Kepler telescope, several studies have confirmed the light-curve modulations produced by extrasolar planets (e.g. Borucki et al. 2009; Shporer et al. 2011; Quintana et al. 2013). The modelling of these modulations can provide the mass of the bound object, which makes it an alternative method with which to confirm its planetary nature.

Lillo-Box et al. (2014b) used this photometric technique (together with a careful characterisation of the host star) to establish the planetary nature of the object transiting the star Kepler-91 (KOI-2133 or KIC 8219268). It was detected by the Kepler mission (Borucki et al. 2011) and announced as a planet candidate in the second release of the mission on February 2012 (Batalha et al. 2013). The exhaustive analysis of the light-curve modulations, transit dim, and asteroseismic signals performed by Lillo-Box et al. (2014b) led us to classify this planet as an inflated hot Jupiter $\left(M_{\mathrm{p}}=0.88_{-0.33}^{+0.17}\right.$ and $\left.R_{\mathrm{p}}=1.384_{-0.054}^{+0.011}\right)$ that orbits very close $\left(r=2.32_{-0.22}^{+0.07} R_{\star}\right.$ at periastron passage) to a K3 III giant star $\left(M_{\star}=1.31 \pm 0.10 M_{\odot}, R_{\star}=6.30 \pm 0.16 R_{\odot}\right)$.
However, two studies (Esteves et al. 2013; Sliski \& Kipping 2014) claimed a possible non-planetary nature for this system. Both papers suggested that it might instead be a self-luminous (and thus star-like) object. Based on transit-fitting analysis and a claimed detection of the secondary eclipse, Esteves et al. (2013) concluded that the object emits light so they rejected it as a planet. In contrast, Angerhausen et al. (2014) provided a high-level analysis of the entire light-curve (encompassing all available Kepler observations). They detected several dimmings in the phase-folded light-curve, achieved similar conclusions as Lillo-Box et al. (2014b), and suggested that the detected dimmings are poorly explained with a secondary eclipse alone. Sliski \& Kipping (2014) estimated (also with transit-fitting) the mean stellar density of the star and compared it with the asteroseismic determination. The significant difference found between the two values was attributed to the non-planetary nature of the transiting object.

Therefore, the problem is still open. In this paper, we provide additional and independent confirmation of the planetary nature of Kepler-91b.

\section{Observations and data analysis}

\subsection{Observations and basic reduction}

High-resolution spectra were collected during the Kepler observing window of May-July 2012 with the Calar Alto Fiber-fed Echelle spectrograph (CAFE, Aceituno et al. 2013), mounted on the $2.2 \mathrm{~m}$ telescope in Calar Alto Observatory (Almería, Spain). The instrument provides an average spectral resolution of around $\lambda / \Delta \lambda=63000$ across the whole spectral range between $4000 \AA$ and $9500 \AA$, allowing nominal radial velocity accuracies at the level of few tens of meters per second for FGK stars. 


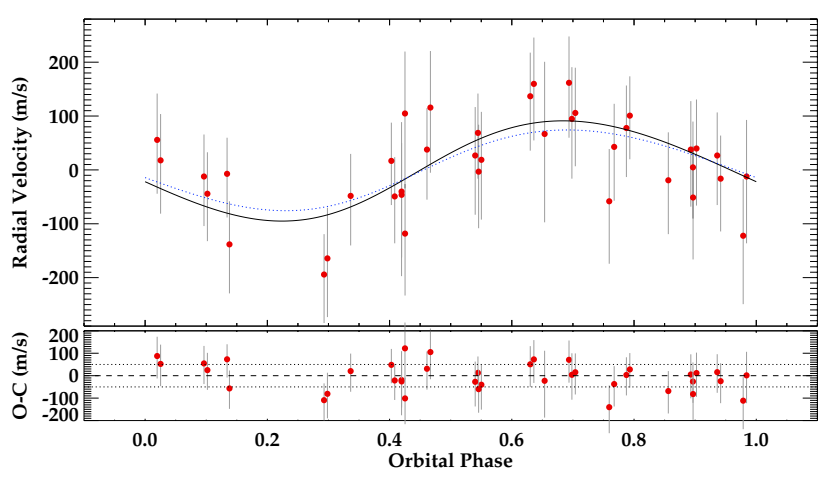

Fig. 1. Radial velocity data (red circles). The solid black line shows the fit to the acquired radial velocity data by assuming the period obtained by the Kepler team (Batalha et al. 2013) and the small eccentricity derived in Lillo-Box et al. (2014b) using the light-curve modulations (REB). The dotted line represents the independent curve obtained by using the parameters extracted from Lillo-Box et al. (2014b), using the REB modulations.

The exposure time for Kepler-91, a $m_{\text {Kep }}=12.5$ mag giant star (spectral type K3 III), was set between 1800 and $2700 \mathrm{~s}$, depending on the weather conditions (seeing, atmospheric transparency, etc.). We typically obtained from two to three spectra per night for this object. The signal-to-noise ratio $(\mathrm{S} / \mathrm{N})$ was calculated as the inverse of the root mean square of the spectra over a continuum region without lines centred at $6500 \AA$ (where the efficiency peak of CAFE is located).

In total, 40 spectra were acquired in 20 nights, with a median $\mathrm{S} / \mathrm{N}$ of 11 . Continuum and bias images were obtained for reduction purposes and thorium-argon (ThAr) arcs were acquired after each science spectrum to calibrate the wavelength accurately. All images were processed with the pipeline provided by the observatory (see Aceituno et al. 2013, for further details on the reduction process). It uses a specific ThAr line list of several hundred features to achieve wavelength calibration with a precision at the $1 \mathrm{~m} / \mathrm{s}$ level. Every spectrum was wavelength-calibrated with the immediate arc obtained just after the science observation at the same telescope position. The data presented here were taken during the earlier periods of operations of the instrument, when the thermal and vibrational control systems were still not fully operational.

\subsection{Extracting the radial velocity with GAbox}

We applied an active cross-correlation method to extract the radial velocity information of the reduced spectra. Zucker (2003), it is showed that maximum-likelihood parameter determination is equivalent to cross-correlation (see Sect. 2 in that paper). The method assumes that the observed spectrum, $f(n)$, can be modelled by a template, $g(n)$, scaled by a constant $\left(a_{0}\right)$, shifted by a determined number of bins $\left(s_{0}\right)$ with the addition of random white Gaussian noise with a specific standard deviation $\left(\sigma_{0}\right)$, $f(n)=a_{0} g\left(n-s_{0}\right)+\mathcal{N}\left(0, \sigma_{0}^{2}\right)$. Thus, the natural logarithm of the likelihood function becomes

$\log L=-N \log \sigma_{0}-\frac{1}{2 \sigma_{0}^{2}} \sum_{n}\left[f(n)-a_{0} g\left(n-s_{0}\right)\right]^{2}+C$,

where $N$ is the total number of bins (pixels) of the spectrum and $C$ is a constant independent of the parameters. The set of parameters $[\hat{s}, \hat{a}, \hat{\sigma}]$ maximising the function provides the best fit of the modified template to the observations. In particular, $\hat{s}$ can be identified with the radial velocity of the star. This method assumes zero mean for both the template and the observed spectrum, therefore we subtracted their corresponding means.

We used a modified version of our genetic algorithm that we presented in Lillo-Box et al. (2014b; GAbox), to find the set of parameters maximising the likelihood in Eq. (1). We used a synthetic spectrum from Coelho et al. (2005) of the same spectral type as Kepler-91 as a template. This improved algorithm searches for the best-fit set of parameters with no need of exploring the whole parameter space (see general details of the method in Lillo-Box et al. 2014b). In this case, the routine first uses large step sizes of $5 \mathrm{~km} \mathrm{~s}^{-1}$ to search for the rough region in which the maximum is located. When this region is found, the step sizes start to decrease gradually (until the $1 \mathrm{~m} / \mathrm{s}$ level) each time the algorithm finds a maximum in the likelihood function. After the final maximum has been found, the process is repeated $G$ times, with $G$ being the number of super-generations provided by the user (typically $G>20$ ). The final radial velocity of a specific order $(\hat{s})$ is obtained as the median of the $G$ convergence values. The upper and lower confidence levels of each $G_{i}$ value correspond to the $3 \sigma$ statistical uncertainty. The final uncertainty of the calculated RV of the order $\left(\sigma_{\hat{s}}\right)$ is computed by bootstrapping all $G$ values with their corresponding uncertainties.

We determined one shift per order ( 84 in total for CAFE) and combined them to obtain the RV of the star. The final value and its uncertainty were thus computed by the median of all orders and bootstrapping the individual results to obtain $3 \sigma$ errors.

\section{Results}

Table 1 summarises the observing characteristics (Julian date, exposure time, $\mathrm{S} / \mathrm{N}$, and phase) as well as the RV values for each epoch obtained as explained in Sect. 2. In Fig. 1, we show the phase-folded RV data.

Prior to fitting a Keplerian orbit to the RV data, we performed a Lomb-Scargle periodogram (Fig. 2) to check whether we detect the planetary signal at the expected period $(T=$ $6.246580 \pm 0.000082$ days as determined by Batalha et al. 2013). We restricted the period search to a specific range. The longest period explored was set to the longest time span between our observations (i.e. $T_{\max }=t_{\max }-t_{\min }=62$ days, where $t_{\max }$ and $t_{\min }$ are the earliest and latest Julian dates in our observations). Since the observations are unevenly separated, the shortest period searched was set to the median of the inverse time interval between data points, as was proposed by Debosscher et al. (2007) and Ivezić et al. (2013), $T_{\min }=\overline{\Delta t}=2.8$ days $^{1}$. The significant peak in the power spectrum (with a false-alarm probability $^{2}$ of FAP $=0.09 \%$, over the $0.1 \%$ level) coincides with the expected period of the planet. This provides clear confirmation for the detection of a periodic signal. Consequently, we can affirm that we are detected the RV signal of Kepler-91b.

We then used the RVLIN software 3 (Wright \& Howard 2009) and its additional package BOOTTRAN for parameter uncertainties estimation with bootstrapping (described in Wang et al. 2012) to fit our RV data to a Keplerian orbital solution. Since we have extensive observations of its transit, the period of the transiting object can be far more accurately determined by the transit analysis. Thus, we decided to fix the period to that provided by Batalha et al. (2013). Because of the relatively large

1 Eyer \& Bartholdi (1999) claimed that for most practical cases, lower periodicities (higher frequencies) can be detected even for strongly (but randomly) under-sampled observations.

2 Calculated by using the astroML python module (Vanderplas et al. 2012) and its bootstrapping package.

http://exoplanets.org/code/ 
J. Lillo-Box et al.: Radial velocity confirmation of Kepler-91 b

Table 1. Observational data and determined radial velocity. Julian date is calculated at mid-observation.

\begin{tabular}{|c|c|c|c|c|c|c|c|c|c|c|c|}
\hline $\begin{array}{c}\text { Julian date } \\
\text { (days)-2456000 }\end{array}$ & $\overline{S / N}$ & Phase & $\begin{array}{c}\mathrm{RV} \\
\left(\mathrm{km} \mathrm{s}^{-1}\right)\end{array}$ & $\begin{array}{c}\text { Julian date } \\
\text { (days) }-2456000\end{array}$ & $\overline{S / N}$ & Phase & $\begin{array}{c}\mathrm{RV} \\
\left(\mathrm{km} \mathrm{s}^{-1}\right)\end{array}$ & $\begin{array}{c}\text { Julian date } \\
\text { (days)-2456000 }\end{array}$ & $\overline{S / N}$ & Phase & $\begin{array}{c}\mathrm{RV} \\
\left(\mathrm{km} \mathrm{s}^{-1}\right)\end{array}$ \\
\hline 079.3736990 & 10.2 & 0.6936 & $-61.849_{-0.086}^{+0.102}$ & 095.6096755 & 10.7 & 0.2928 & $-62.205_{-0.075}^{+0.090}$ & 116.4939625 & 11.3 & 0.6361 & $-61.851_{-0.086}^{+0.105}$ \\
\hline 079.4383387 & 11.6 & 0.7039 & $-61.905_{-0.084}^{+0.099}$ & 096.4018846 & 10.3 & 0.4196 & $-62.051_{-0.129}^{+0.097}$ & 121.4241545 & 11.0 & 0.4253 & $-62.129_{-0.092}^{+0.0115}$ \\
\hline 080.6188492 & 10.0 & 0.8929 & $-61.973_{-0.090}^{+0.084}$ & 096.4367742 & 9.6 & 0.4252 & $-61.906_{-0.115}^{+0.129}$ & 123.5107198 & 10.8 & 0.7594 & $-62.069_{-0.097}^{+0.092}$ \\
\hline 080.6408499 & 9.5 & 0.8964 & $-62.006_{-0.085}^{+0.095}$ & 099.3819275 & 10.7 & 0.8966 & $-62.062_{-0.095}^{+0.115}$ & 123.5616251 & 11.0 & 0.7675 & $-61.968_{-0.080}^{+0.100}$ \\
\hline 092.4534211 & 11.3 & 0.7875 & $-61.933_{-0.079}^{+0.091}$ & 102.5440124 & 11.4 & 0.4029 & $-61.994_{-0.071}^{+0.082}$ & 128.4226132 & 11.1 & 0.5457 & $-62.014_{-0.087}^{+0.105}$ \\
\hline 092.4885156 & 11.5 & 0.7931 & $-61.910_{-0.073}^{+0.081}$ & 102.5786588 & 11.6 & 0.4084 & $-62.060_{-0.073}^{+0.087}$ & 131.3837431 & 11.4 & 0.0197 & $-61.955_{-0.086}^{+0.100}$ \\
\hline 093.3820328 & 11.1 & 0.9361 & $-61.984_{-0.080}^{+0.092}$ & 103.4294963 & 11.6 & 0.5446 & $-61.942_{-0.073}^{+0.005}$ & 131.4186423 & 11.5 & 0.0253 & $\begin{array}{r}-01.993_{-0.086}^{+0.086} \\
-096\end{array}$ \\
\hline 093.4167639 & 11.4 & 0.9417 & $-62.027_{-0.080}^{+0.098}$ & 103.4647180 & 10.6 & 0.5502 & $-61.992_{-0.089}^{+0.111}$ & 140.3877688 & 11.0 & 0.4612 & $-61.973_{-0.077}^{+0.090}$ \\
\hline 094.3825217 & 11.4 & 0.0963 & $-62.023_{-0.078}^{+0.090}$ & 111.6216095 & 10.9 & 0.8561 & $-62.030_{-0.089}^{+0.100}$ & 140.4227569 & 10.8 & 0.4668 & $-61.895_{-0.105}^{+0.121}$ \\
\hline 094.4174484 & 11.6 & 0.1019 & $-62.055_{-0.077}^{+0.088}$ & 112.3867977 & 11.4 & 0.9786 & $-62.133_{-0.105}^{+0.127}$ & 141.5906808 & 10.6 & 0.6537 & $-61.944_{-0.134}^{+0.164}$ \\
\hline 094.6184378 & 10.6 & 0.1341 & $-62.018_{-0.067}^{+0.081}$ & 112.4215921 & 11.5 & 0.9841 & $-62.023_{-0.105}^{+0.124}$ & & & & \\
\hline
\end{tabular}

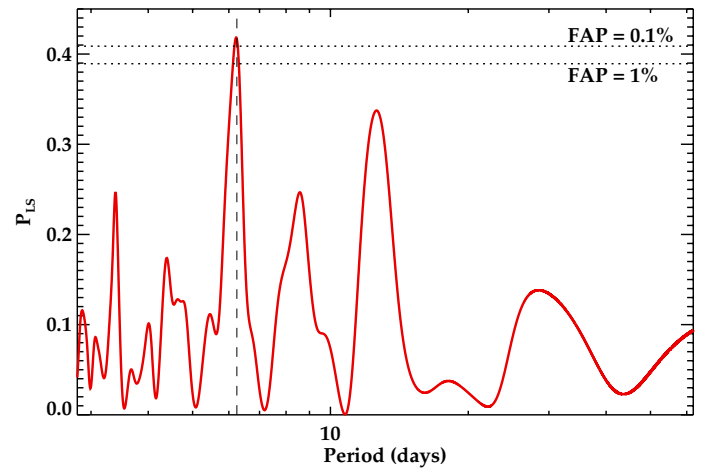

Fig. 2. Lomb-Scargle periodogram of the radial velocity data obtained with CAFE. The dotted lines show the false-alarm probability levels of $\mathrm{FAP}=0.1 \%$ and FAP $=1 \%$. The vertical dashed line shows the period derived by transit detection. The detected peak at $6.23 \pm 0.03$ days in this $\mathrm{RV}$ periodogram has an $\mathrm{FAP}=0.09 \%$.

uncertainties in the RV and incomplete coverage of the RV curve, we also decided to fix the eccentricity of the orbit to the slightly non-circular value determined by Lillo-Box et al. (2014b), $e=0.066_{-0.017}^{+0.013}$. The free parameters for this fitting were the semi-amplitude of the RV variations $(K)$, the systemic velocity of the system $\left(V_{\text {sys }}\right)$, and the orbital argument of the periastron $(\omega)$.

We used the asteroseismic determination of the stellar mass of the host star by Lillo-Box et al. $(2014 \mathrm{~b}), M_{\star}=1.31 \pm 0.1 M_{\odot}$, to obtain an accurate value of the minimum mass of the transiting object (i.e $M_{\mathrm{p}} \sin i$ ). Moreover, we know that the orbit of this planet is highly inclined with respect to our line of sight. The inclination was also provided by Lillo-Box et al. (2014b) $\left(i=68.5_{-2.0}^{+1.0}\right.$ degrees $)$ and is supported by previous light-curve analysis such as Tenenbaum et al. (2012), who derived $i=71.4 \pm 2.5$ degrees, in good agreement with our value. Thus, we can directly determine the absolute mass of the orbiting object.

The results of the RVLIN fitting process are shown in Table 2, the fitted model is plotted in Fig. 1. We investigated the significance of that fit against a constant model (which would imply that we are just detecting noise). We infer a Bayesian information criterion $\left(\mathrm{BIC}^{4}\right)$ for the constant model and for the

$4 \mathrm{BIC}=\chi^{2}+k \log N$, where $\chi^{2}=\sum \frac{\left(\mathrm{RV}_{\text {model }}-\mathrm{RV}_{\mathrm{obs}}\right)^{2}}{\sigma_{\mathrm{RV}}^{2}}, k$ is the number of free parameters, and $N$ is the number of data points.
Table 2. Best-fit and derived parameters from the RV analysis.

\begin{tabular}{rccc}
\hline \hline Parameter & Value & Units & Assumptions $^{a}$ \\
\hline$K$ & $93 \pm 17$ & $\mathrm{~m} / \mathrm{s}$ & $e(\mathrm{REB}), P(\mathrm{TR})$ \\
$V_{\text {sys }}$ & $-62.011 \pm 0.011$ & $\mathrm{~km} \mathrm{~s}^{-1}$ & $e(\mathrm{REB}), P(\mathrm{TR})$ \\
$\omega$ & $250 \pm 100$ & $\mathrm{deg}$. & $e(\mathrm{REB}), P(\mathrm{TR})$ \\
$M_{\mathrm{p}} \sin i$ & $1.01 \pm 0.18$ & $M_{\text {Jup }}$ & $e(\mathrm{REB}), P(\mathrm{TR}), M_{\star}(\mathrm{AS})$ \\
$a$ & $0.0726 \pm 0.0019$ & $\mathrm{AU}$ & $e(\mathrm{REB}), P(\mathrm{TR}), M_{\star}(\mathrm{AS})$ \\
\hline$M_{\mathrm{p}}$ & $1.09 \pm 0.20$ & $M_{\text {Jup }}$ & $i(\mathrm{TR})$ \\
$\rho_{\mathrm{p}}$ & $0.41_{-0.08}^{+0.13}$ & $\rho_{\text {Jup }}$ & $R_{\mathrm{p}}(\mathrm{TR})$ \\
$a / R_{\star}$ & $2.48 \pm 0.12$ & & $R_{\star}(\mathrm{AS})$ \\
\hline
\end{tabular}

Notes. Parameters below the horizontal line are derived from those above. ${ }^{(a)}$ Assumed parameters and the method used to determine them. Parameters: $e$ (eccentricity), $P$ (period), $M_{\star}$ (stellar mass), $i$ (orbital inclination), $R_{\mathrm{p}}$ (planet radius), and $R_{\star}$ (stellar radius). Methods: $\mathrm{AS}=$ asteroseismology, $\mathrm{TR}=$ transit fitting, $\mathrm{REB}=$ light-curve modulations fitting. Assumed values are taken from Lillo-Box et al. (2014b).

$\mathrm{RV}$ model of $\mathrm{BIC}_{\text {const. }}=30.5$ and $\mathrm{BIC}_{R V}=27.8^{5}$. This implies a $\triangle \mathrm{BIC}=2.7$, which provides positive (although not strong) evidence for the RV model (positive detection) against the constant model (negative detection). Alternatively, we obtain a value of 12.7 for an F-test with weighted residuals ${ }^{6}$. This value is higher than the corresponding value of the F-distribution for a $99 \%$ confidence level, $F_{0.01}\left(p_{2}-p_{1}, N-p_{2}\right)=F_{0.01}(3,36)=4.38$. Thus, we can confirm that the detected RV variability is significant at $99 \%$ confidence level with respect to pure noise.

The radial velocity data confirm the Jupiter-like mass $\left(M_{\mathrm{p}} \sin i=1.01 \pm 0.18 M_{\text {Jup }}\right)$ of the object orbiting Kepler-91. Using our previous value for the inclination, the absolute mass becomes $M_{\mathrm{p}}=1.09 \pm 0.20 M_{\mathrm{Jup}}$. This result agrees well, within the uncertainties, with the derived mass in the confirmation paper of Kepler-91b (Lillo-Box et al. 2014b), $M_{\mathrm{p}}=0.88_{-033}^{+0.17} M_{\text {Jup }}$. Moreover, the semi-major axis $a_{\mathrm{RV}}=0.0726 \pm 0.0019 \mathrm{AU}$ also agrees extremely well with the value determined by the light-curve modulations in Lillo-Box et al. (2014b) of $a_{\mathrm{REB}}=$ $0.072_{-0.007}^{+0.002} \mathrm{AU}$. The corresponding radial velocity model using the photometrically derived parameters obtained by Lillo-Box et al. (2014b) is also plotted in Fig. 1 for comparison purposes.

\footnotetext{
5 Assuming a circular orbit, we obtain $\mathrm{BIC}_{\mathrm{RV}(e=0)}=28.3$.

${ }_{6} F=\frac{\chi_{1}^{2}-\chi_{2}^{2}}{p_{2}-p_{1}} / \frac{\chi_{2}^{2}}{N-p_{2}}$, where $p_{1}$ and $p_{2}$ are the free parameters of both models (so that $p_{2}>p_{1}$ ), and $N$ the number of data points.
} 


\section{Discussion and conclusions}

The fit to the RV data provides orbital parameters that agree excellently well with those obtained by the photometric analysis provided in the confirmation paper ${ }^{7}$. Thus, we present independent support of the planetary-mass of the object transiting Kepler-91. Moreover, the transit and asteroseismic analysis yield a radius of the transiting object of $R_{\mathrm{p}}=1.384_{-0.054}^{+0.011} R_{\text {Jup }}$, providing a mean density of $\rho_{\mathrm{p}}=0.33 \pm 0.08 \rho_{\text {Jup }}$.

These results disagree with Esteves et al. (2013) and Sliski \& Kipping (2014). The former was already discussed in Lillo-Box et al. (2014b), where several arguments were given against the proposed self-luminous scenario for Kepler-91b. In brief, Esteves et al. (2013) derived a mass for the transiting object of $5.92_{1.12}^{+0.68} M_{\text {Jup }}$ and obtained discrepant day/night-side and equilibrium temperatures based on a claimed detection of the secondary eclipse. However, as we noted and Angerhausen et al. (2014) concluded as well, the detection of the secondary eclipse is neither clear nor conclusive. Moreover, given their derived mass for this companion with a Jupiter-like radius, it is difficult to explain their proposed stellar nature. These types of objects are only found in very young stellar associations (with ages younger than $10 \mathrm{Myr}$ ) such as Collinder 69 or $\sigma$-Orionis. Thus, even assuming their derived higher mass, this proposed falsepositive configuration can be ruled out.

The alternative explanation provided by Esteves et al. (2013) suggests that the system is actually an eclipsing binary diluted by a contaminating third stellar source, a foreground or background star. However, Lillo-Box et al. (2012, 2014a) provided high-resolution images of this system (among other Kepler candidates) to rule out this and other configurations. According to Sect. 4.1 .2 of the latter paper (based on Law et al. 2013), the planetary transit of Kepler-91b cannot be mimicked by the presence of a diluted star fainter than the transited star. Only a blended source with a very small radius that is brighter than the transited star can dilute the binary eclipse and mimic a planetary transit. However, in the high-resolution image obtained by Lillo-Box et al. (2014a) we did not find companions farther away than 0.1 arcsec. Thus, the probability for an undetected chancealigned source brighter than 12.5 magnitudes and closer than 0.1 arcsec is lower than $10^{-6}$.

Sharing the opinion of Esteves et al. (2013), Sliski \& Kipping (2014) also classified Kepler-91b as a false positive. They compared the asteroseismic determination of the stellar density $\rho_{\star \text {,astero }}=6.81 \pm 0.32 \mathrm{~kg} / \mathrm{m}^{3}$ (derived by Huber et al. 2013 ) with the observed value $\rho_{\star, \mathrm{obs}}=43.47_{-3.35}^{+0.67} \mathrm{~kg} / \mathrm{m}^{3} \mathrm{de}-$ rived by them directly from transit fitting. Based on the large discrepancy between the two values, they concluded that to explain this disagreement, the orbit of the planet would have to be highly eccentric such that the planet is essentially expected to be in-contact with the star. Basically, their derived observed stellar density corresponds to a semi-major axis of the companion of $a / R_{\star}=4.476_{-0.118}^{+0.023}$, similar to that of Esteves et al. (2013), $a / R_{\star}=4.5$. The authors claimed that the two determinations of the semi-major axis are independent, but they both used the same set of photometric data and the same observational effect (the transit signal).

Instead, we have determined the semi-major axis of the orbit by using three truly independent observational effects, namely the transit fitting $\left(a / R_{\star}\right)_{\text {transit }}=2.40 \pm 0.12$ (also supported by the previous analysis of Tenenbaum et al. 2012), light-curve

7 The agreement between the ellipsoidal modulation mass and the RV mass has already been demonstrated in systems such TrES-2 or HAT-P-7 (see Faigler \& Mazeh 2014). modulations in the out-of-transit region $\left(a / R_{\star}\right)_{\mathrm{REB}}=2.45_{-0.30}^{+0.15}$, and the current radial velocity analysis $\left(a / R_{\star}\right)_{\mathrm{RV}}=2.48 \pm 0.12$. All three estimations provide autonomous and coincident measurements of the semi-major axis. As stated by Sliski \& Kipping (2014) and already pointed out in Lillo-Box et al. (2014b), this lower value implies an observed stellar density that agrees excellently with the asteroseismic analysis, thus confirming the planetary nature of the object orbiting Kepler-91 and rejecting the self-luminous scenario. Possible explanations for the large discrepancy of the other determinations of $a / R_{\star}$ might involve some assumptions when fitting the transit. They both assumed i) a circular orbit, while we found that the shape of the ellipsoidal variations need a non-zero - although low- eccentricity; and ii) a spherical shape for the host star, which does not apply here since we clearly find light-curve modulations due to deformations of the stellar atmosphere.

The results presented here, together with the original confirmation paper (Lillo-Box et al. 2014b), provide strong evidence for the planetary-nature of Kepler-91b. Finally, this is the first planet confirmed from CAHA using CAFE, and we have proved the capability of this instrument for this type of research.

Acknowledgements. This research has been funded by Spanish grant AYA201238897-C02-01. J.L.-B. thanks the CSIC JAE-predoc programme for the Ph.D. fellowship. We also thank CAHA for allocating our observing runs. P.F. and N.C.S. acknowledge support by Fundação para a Ciência e a Tecnologia (FCT) through Investigador FCT contracts of reference IF/01037/2013 and IF/00169/2012, respectively, and POPH/FSE (EC) by FEDER funding through the program "Programa Operacional de Factores de Competitividade COMPETE". We also acknowledge the support from the European Research Council/European Community under the FP7 through Starting Grant agreement number 239953

\section{References}

Aceituno, J., Sánchez, S. F., Grupp, F., et al. 2013, A\&A, 552, A31 Angerhausen, D., DeLarme, E., \& Morse, J. A. 2014, ApJ, submitted [arXiv: 1404.4348]

Batalha, N. M., Rowe, J. F., Bryson, S. T., et al. 2013, ApJS, 204, 24 Borucki, W. J., Koch, D., Jenkins, J., et al. 2009, Science, 325, 709 Borucki, W. J., Koch, D. G., Basri, G., et al. 2011, ApJ, 736, 19 Coelho, P., Barbuy, B., Meléndez, J., Schiavon, R. P., \& Castilho, B. V. 2005, A\&A, 443, 735

Debosscher, J., Sarro, L. M., Aerts, C., et al. 2007, A\&A, 475, 1159

Esteves, L. J., De Mooij, E. J. W., \& Jayawardhana, R. 2013, ApJ, 772, 51

Eyer, L., \& Bartholdi, P. 1999, A\&AS, 135, 1

Faigler, S., \& Mazeh, T. 2014, ApJ, submitted [arXiv: 1407.2361]

Gould, A., \& Loeb, A. 1992, ApJ, 396, 104

Holman, M. J., Fabrycky, D. C., Ragozzine, D., et al. 2010, Science, 330, 51

Huber, D., Chaplin, W. J., Christensen-Dalsgaard, J., et al. 2013, ApJ, 767, 127

Ivezić, Ż., Connolly, A., VanderPlas, J., \& Gray, A. 2013, Statistics, Data Mining, and Machine Learning in Astronomy (Princeton University Press) Law, N. M., Morton, T., Baranec, C., et al. 2013, ApJ, submitted [arXiv: 1312.4958]

Lazorenko, P. F., Sahlmann, J., Ségransan, D., et al. 2011, A\&A, 527, A25

Lillo-Box, J., Barrado, D., \& Bouy, H. 2012, A\&A, 546, A10

Lillo-Box, J., Barrado, D., \& Bouy, H. 2014a, A\&A, 566, A103

Lillo-Box, J., Barrado, D., Moya, A., et al. 2014b, A\&A, 562, A109

Mao, S., \& Paczynski, B. 1991, ApJ, 374, L37

Mayor, M., \& Queloz, D. 1995, Nature, 378, 355

Muterspaugh, M. W., Lane, B. F., Kulkarni, S. R., et al. 2010, AJ, 140, 1657

Quintana, E. V., Rowe, J. F., Barclay, T., et al. 2013, ApJ, 767, 137

Santerne, A., Díaz, R. F., Moutou, C., et al. 2012, A\&A, 545, A76

Shporer, A., Jenkins, J. M., Rowe, J. F., et al. 2011, AJ, 142, 195

Sliski, D. H., \& Kipping, D. M. 2014, ApJ, accepted [arXiv: 1401 . 1207]

Tenenbaum, P., Jenkins, J. M., Seader, S., et al. 2012, ApJS, submitted [arXiv: 1212.2915]

Vanderplas, J., Connolly, A., Ivezić, Ž., \& Gray, A. 2012, in Conf. on Intelligent Data Understanding (CIDU), 47

Wang, S. X., Wright, J. T., Cochran, W., et al. 2012, ApJ, 761, 46

Wright, J. T., \& Howard, A. W. 2009, ApJS, 182, 205

Zucker, S. 2003, MNRAS, 342, 1291 